\title{
Incremental Diversity: A Framework for Rate-Adaptation/Energy-Conservation Enhancement in MIMO Systems
}

\author{
Tallal Elshabrawy, Sally Nafie \\ Faculty of Information Engineering and Technology, the German University in Cairo, Cairo, Egypt \\ Email: tallal.el-shabrawy@guc.edu.eg, sally.mahmoud@guc.edu.eg
}

Received 30 June 2014; revised 20 July 2014; accepted 10 August 2014

Copyright (c) 2014 by authors and Scientific Research Publishing Inc.

This work is licensed under the Creative Commons Attribution International License (CC BY). http://creativecommons.org/licenses/by/4.0/

\begin{abstract}
In recent years, MIMO technology has emerged as one of the technical breakthroughs in the field of wireless communications. Two famous MIMO techniques have been investigated thoroughly throughout the literature; Spatial Multiplexing, and Space Time Block Coding. On one hand, Spatial Multiplexing offers high data rates. On the other hand, Space Time Block Coding presents transmission fidelity. This imposes a fundamental tradeoff between capacity and reliability. Adaptive MIMO Switching schemes have been proposed to select the MIMO scheme that best fits the channel conditions. However, the switching schemes presented in the literature directly switch between the MIMO endpoints. In this paper, an adaptive MIMO system that incrementally switches from multiplexing towards diversity is proposed. The proposed scheme is referred to as incremental diversity and can be set to operate in two different modes; Rate-Adaptive, and Energy-Conservative Incremental Diversity. Results indicate that the proposed incremental diversity framework achieves transmission reliability offered by MIMO diversity, while maintaining a gradual increase in spectral efficiency (in the Rate-Adaptive mode) or a reduction in required number of received symbols (in the Energy-Conservative mode) with increase in the SNR.
\end{abstract}

\section{Keywords}

MIMO Diversity-Multiplexing Tradeoff, Hybrid Spatial Multiplexing-Space Time Block Coding, Incremental Redundancy, Energy-Conservative MIMO, Rate-Adaptive MIMO

\section{Introduction}

In recent years wireless communications have witnessed a rapid increase in the demand for capacity driven by 
cellular mobile, Internet, and multimedia services [1]. However, increasing the capacity requires a direct increase in available radio spectrum which is on the other hand limited. Multiple Input Multiple Output (MIMO) systems are among the primary techniques called upon to enhance spectral efficiency in order to meet required capacity demands of wireless communication systems under the restricted radio spectrum resources. MIMO systems are characterized by the deployment of multiple antennas at both the transmitter and the receiver sides. MIMO systems are of superior performance because they offer array, diversity, and multiplexing gains [2].

The MIMO schemes can be categorized into two famous approaches: Spatial Multiplexing (SM), and Space Time Block Coding (STBC). On one hand, SM offers high data rates [3]. On the other hand, STBC presents transmission fidelity [4]. This actually imposes a fundamental tradeoff between the two extremes; capacity and reliability [5]. A major concern is devoted to considering the switching between rate and reliability, or namely SM, and STBC. In [6], a switching criterion is derived based on the channel state information. Given a fixed bit rate, the scheme that offers the least probability of error is decided on at the receiver side, and fed back to the transmitter. The switching criterion in [7] is formulated the other way around. At a certain predefined outage probability, the scheme that renders higher spectral efficiency is chosen. Another perspective is introduced in [8], where Hybrid SM/STBC is presented as a MIMO technology that combines SM and STBC, and hence, compromises between the advantages of the two schemes.

Incremental Redundancy (IR) is a Hybrid Automatic Repeat Request (HARQ) scheme adopted for error control in wireless networks [9] [10]. The key feature is that, upon receiving a negative acknowledgement (NACK) indicating an erroneous packet, the transmitter transmits incremental redundancy symbols that aim at enforcing the robustness of the data previously transmitted [11]. The process is repeated iteratively until an acknowledgement (ACK) is received, or the max number of retransmissions is reached.

This paper adopts the concept of incremental redundancy within MIMO systems. The proposed system incrementally switches from SM, towards STBC or hybrid SM/STBC. The system is initialized with a pure multiplexing mode. An error detection check is performed after decoding and successful detection is flagged via an acknowledgement. A negative acknowledgement due to false detection triggers the system to incrementally switch towards the diversity mode where it combines a part of the diversity symbols constituting a composite multiplexing-diversity mode. The incremental diversity symbols are combined until a positive-acknowledgment is instigated or the system exhausts its full diversity capabilities.

The above concept of incremental diversity has been introduced in our work in [12]. In this paper, the proposed incremental diversity scheme can be set to operate in two different modes; Rate-Adaptive, and EnergyConservative. In the rate-adaptive mode, the results of [12] are extended to show that the proposed scheme enhances spectral efficiency while maintaining BER reliability under various MIMO configurations. In particular, it is shown that at certain SNR operation ranges, the spectral efficiency of lower order constellation sizes approaches or even surpasses those of higher order constellations. This is achieved while maintaining BER superiority of lower constellation sizes along with a significant reduction in decoding complexity. In the energy-conservative mode on the other hand, the results demonstrate that the proposed incremental diversity MIMO deployed within a broadcast communication environment has the potential to contribute to significant reduction in symbol-level reception energy requirements since each receiver has the ability to adapt the required number of symbols for decoding based on its instantaneous experienced Signal-to-Noise ratio.

The rest of this paper is organized as follows. In Section 2, the different MIMO schemes including SM, STBC, and Hybrid SM/STBC are reviewed within the context of the system model. The proposed Incremental Diversity (ID) scheme along with its two modes of operation is introduced in Section 3. Performance of the proposed scheme in terms of error performance, decoding complexity, spectral efficiency, and energy conservation is evaluated in Section 4 through Monte Carlo simulations. Finally, Section 5 presents the conclusion and future work. Throughout the paper, boldface lowercase letters indicate vectors, while boldface uppercase ones indicate matrices. Moreover, ()$^{*},()^{\mathrm{T}}$, and ()$^{\mathrm{H}}$ are used to denote the complex conjugate, transpose, and conjugate transpose, respectively.

\section{System Model}

A typical MIMO communication system consists of $n_{t}$ transmit antennas, and $n_{r}$ receive antennas. Modulated symbols are fed into a space-time encoder, and converted into space-time symbols. The transmission is described by an $n_{t} \times p$ transmission matrix where symbols are transmitted from $n_{t}$ Tx antennas in $p$ time slots. These symbols are transmitted over a MIMO channel, and received by the antenna array deployed at the receiver side. 
Assuming perfect Channel State Information (CSI) at the receiver side, the space time decoder estimates the modulated symbols that were intended to be transmitted. The system can be described by

$$
\boldsymbol{R}=\frac{1}{\sqrt{E_{s} n_{t}}} \boldsymbol{H} \boldsymbol{X}+\boldsymbol{N}
$$

where $E_{s}$ is the average energy per modulated symbol, $\boldsymbol{X} \in \mathbb{C}^{n_{t} \times p}$ is the $n_{t} \times p$ space time matrix transmitted from $n_{t}$ transmit antennas in $p$ time slots, $\boldsymbol{H} \in \mathbb{C}^{n_{r} \times n_{t}}$ is the $n_{r} \times n_{t}$ Rayleigh channel matrix with entries; $h_{j i}$, representing the fading coefficient from transmit antenna $i$ to receive antenna $j, \quad \boldsymbol{N} \in \mathbb{C}^{n_{r} \times p}$ is the additive white Gaussian noise matrix with zero mean and variance $N_{0} / 2$, and $\boldsymbol{R} \in \mathbb{C}^{n_{r} \times p}$ is the $n_{r} \times p$ received matrix. In this paper, slowly varying flat fading Rayleigh Model is assumed [6]. According to the processing of the modulated symbols inside the space time encoder, the transmission scheme can be classified into SM, STBC or Hybrid SM/STBC.

\subsection{Spatial Multiplexing (SM)}

SM is a space time coding technique that aims at increasing the data rate but not the robustness of the transmission [13]. The key feature of SM is demultiplexing of a serial symbol stream into parallel substreams [14]. The demultiplexed substreams are then mounted and transmitted simultaneously from $n_{t}$ transmit antennas. During each symbol period, $n_{t}$ independent modulated symbols constituting an $n_{t} \times 1$ space time symbol, are simultaneously transmitted from the $n_{t}$ antennas. Hence, SM can be viewed as a special case of the general MIMO system where $p=1$. The $n_{t} \times 1$ space time symbol $\boldsymbol{X}$ is given by

$$
\boldsymbol{X}=\left[\begin{array}{lll}
s_{1} & \cdots & s_{n_{t}}
\end{array}\right]^{\mathrm{T}}
$$

where $s_{i}, i=1, \cdots, n_{t}$, is the $i^{\text {th }}$ modulated symbol in the $1 \times n_{t}$ vector coming out of the M-ary modulator.

\subsection{Space Time Block Coding (STBC)}

STBC is another variant of MIMO systems that transmits the modulated symbols and their manipulated replicas from multiple transmit antennas at different time slots [6]. The Alamouti scheme is the simplest STBC encoder [1]. It is built up of $n_{t}=2$ transmit antennas. It involves transmission of 2 modulated symbols—and their complex conjugate-from 2 transmit antennas, in 2 time slots. It is the only orthogonal STBC technique that is a full rate (2 symbols in 2 time slots). Going for higher order antenna array, a compromise is made; either to use an orthogonal STBC encoder with a code rate that is less than unity, or to use quasi-orthogonal codes with higher rates at the expense of the bit error rate performance. In this paper, only the simple full rate Alamouti scheme is considered. The generalization to the orthogonal STBC of arbitrary number of antennas is omitted due to lack of space. Consider the Alamouti scheme, the $2 \times 2$ space time symbol at the encoder output is given by

$$
\boldsymbol{X}=\left[\begin{array}{cc}
s_{1} & -s_{2}^{*} \\
s_{2} & s_{1}^{*}
\end{array}\right]
$$

The first column of the STBC transmission matrix is exactly the same as the transmitted vector of an SM system deploying the same number of antennas. The subsequent column represents replicas of the modulated symbols or their complex conjugate. Hence, for a given infrastructure of $n_{t}$ transmit antennas, SM transmits $n_{t}$ modulated symbols in 1 time slot. On the other hand, STBC requires $p$ time slots in order to transmit the $n_{t}$ symbols and their replicas. Consequently, while SM offers higher capacity inherited in the multiplexing gain, STBC renders higher reliability inherited in the diversity gain.

\subsection{Hybrid SM/STBC}

The Hybrid SM/STBC scheme is a composite MIMO system that comprises both SM and STBC, in an attempt to simultaneously grasp the advantages of the two schemes [8]. The hybrid encoder is built up of a serial-to-parallel converter, and $N$ Alamouti STBC blocks of 2 antennas each. The serial to parallel converter first converts a serial stream of $2 N$ modulated symbols into $N$ parallel substreams of 2 symbols each. Every substream is then fed into an Alamouti encoder, producing a $2 \times 2$ STBC block. The space time symbols are then transmitted from 
$n_{t}=2 N$ Tx antennas, and received by $n_{r}=n_{t}$ Rx antennas after propagating through a Rayleigh channel of $n_{r} \times n_{t}$ fading coefficients, and an AWGN channel of $n_{r} \times 2$ white Gaussian noise of zero mean, and variance $N_{0} / 2$. The $2 N \times 2$ hybrid space time symbol can be described by,

$$
\boldsymbol{X}=\left[\begin{array}{llll}
\boldsymbol{X}_{1}^{\mathrm{T}} & \boldsymbol{X}_{2}^{\mathrm{T}} & \cdots & \boldsymbol{X}_{n}^{\mathrm{T}}
\end{array}\right]^{\mathrm{T}}
$$

where $\boldsymbol{X}_{i}$ for $i=1, \cdots, N$, is the $2 \times 2$ STBC output from block $i$.

As aforementioned, the diversity-multiplexing tradeoff has been given a major concern in the last decades. The challenge of deciding on which scheme to be adopted, has been treated in different perspectives; whether to fix the bit rate, and choose the scheme that minimizes the probability of error, or to keep the probability of error under certain threshold, and choose the scheme that maximizes the spectral efficiency. Most of the proposed algorithms, directly switch between the pure multiplexing and diversity extremes. On the other hand, the Hybrid system described above combines SM and STBC on a fixed basis. This means that it is a compromise between rate and reliability. The motivation behind our proposed scheme is driven from the intention to the ultimate adaptation to the channel quality. In other words, unlike the schemes that directly switch between the two extremes, or the hybrid system that does not adapt to the channel conditions, the proposed scheme adaptively switches between the three schemes described above in a gradual manner.

\section{Proposed Incremental Diversity MIMO}

As illustrated in the previous section, a $2 \times 1$ SM vector appears explicitly in the $2 \times 2$ Alamouti STBC matrix along with the diversity vector. Thus, the received SM symbols constitute the first time slot reception of the STBC. Consequently, having STBC data can solely function as either SM or STBC according to the decision of keeping the first column only or simultaneously using the 2 columns, respectively. For a higher order MIMO system, the available MIMO coding techniques are SM and Hybrid SM/STBC with $N=n_{t} / 2$ Alamouti blocks. Similar to the case of $2 \times 2$ MIMO, the $n_{t}$ symbols of the SM vector constitute the first column of the Hybrid matrix.

Assume the scenario of having a packet of size $P_{s}$ modulated symbols. Since an $n_{t} \times n_{r}$ SM sends $n_{t}$ symbols in 1 time slot. The SM data span $P_{s} / n_{t}$ time slots. On the other hand, the $n_{t} \times n_{r}$ STBC (where $n_{t}=n_{r}=2$ ) or the Hybrid SM/STBC (where $n_{t}=n_{r}>2$ ) requires 2 time slots to send the $n_{t}$ modulated symbols. Hence, the STBC or the Hybrid SM/STBC symbols require $2 P_{s} / n_{t}$ time slots through which $P_{s} / n_{t}$ time slots are assigned for the basic SM data while the other $P_{s} / n_{t}$ time slots include the diversity. Adopting the concept of HARQ, diversity symbols are used only when needed, i.e., when the error check indicates an erroneous packet. The key feature of the proposed Incremental Diversity (ID) scheme is that instead of instantaneously switching from SM to STBC or Hybrid SM/STBC, the proposed scheme incrementally switches from SM towards STBC or hybrid SM/STBC.

The methodology of the ID scheme can be explained as follows. The decoding is first initialized with pure SM mode. A successful error detection check evaluated right after detection instigates an ACK that flags correct detection, and thus no further processing of the received packet is required. An error detection results in a NACK that triggers the combining of a column of the diversity symbols with the basic symbols previously decoded constituting a combined multiplexing - diversity mode. The composite data are then decoded and checked for errors. If a NACK is flagged, another diversity column is concatenated. The process is iteratively repeated until an ACK is received or the full diversity capabilities are exhausted, i.e. when all the $P_{s} / n_{t}$ diversity columns are used. The possible number of iterations of the proposed algorithm is $1+\left(P_{s} / n_{t}\right)$. The 1 stands for the pure multiplexing mode where no diversity columns are combined, and the $P_{s} / n_{t}$ represents the possible number of diversity columns.

It is worth mentioning that for the system to be applicable, the assumption of a block fading Rayleigh channel is presumed. I.e., channel fading coefficients are assumed to remain constant over a block of $2 P_{s} / n_{t}$ time slots, which is the time required for the worst case hybrid SM/STBC with $n_{t}$ Tx antennas to transmit a packet of $P_{s}$ modulated symbols. This assumption is legitimate in a slow fading environment. Similar assumptions have also been adopted in the literature, where the scheme proposed in [6] has assumed constant coefficients over 10 symbol durations. The key advantage of the proposed scheme resides in optimizing the consumption of diversity symbols. The scheme omits the redundant diversity and safeguards its usage according to the system requirement. Depending on the system specifications, the proposed scheme has two modes of operation; rate-adaptive, and energyconservative. 


\subsection{Rate-Adaptive Incremental Diversity (RAID)}

The tremendous growth in the demand for capacity motivated by the incessant development of wireless services necessitates further enhancements in the spectral efficiency (SE) of the existing MIMO technologies. This section adopts the utilization of the proposed ID scheme in a rate-adaptive MIMO approach. Unlike the schemes given in the literature (e.g., [6] [7] [15]), the proposed scheme gradually switches from full multiplexing towards diversity in order to guarantee the ultimate reliability offered by the diversity mode while rendering potential for relatively higher levels of SE attributed to the pure multiplexing mode, particularly in the high SNR region. Furthermore, the decoding complexity of the proposed scheme is quite similar to that of the SM. Hence, the deployment of the proposed RAID scheme should have no profound effect on the system complexity. Figure 1 presents the system model of the proposed RAID algorithm. The transmitter is equipped with $N$ Alamouti STBC blocks with 2 Tx antennas each. This representation provides the generic form of transmission including SM, STBC, and Hybrid SM/STBC coding techniques. The Hybrid SM/STBC encoding is split over two encoding blocks; basic and diversity blocks. For an $n_{t} \times n_{r}$ system processing a packet of size $P_{s}$ modulated symbols, the two encoding blocks take in $n_{t}$ modulated symbols at a time. The basic block outputs the first column of the conventional Hybrid SM/STBC block represented by the vertical concatenation of the $n_{t}$ modulated symbols. The diversity block imitates the second column of the conventional Hybrid block constituting the manipulated replicas of the $n_{t}$ modulated symbols. Nevertheless, operation of the diversity block is suspended awaiting the triggering flag ACK that is fed back from the receiver.

The proposed algorithm is inspired from the idea of incremental redundancy. Modulated symbols are divided into packets of length $P_{s}=n N_{t}$ symbols - where $n$ is an integer - that are fed to the basic and diversity Hybrid SM/STBC blocks. The transmission mode is first initialized with the pure SM mode, where the diversity block is deferred. The $P_{s} / n_{t}$ basic columns are transmitted from the $n_{t}$ Tx antennas. The basic received vectors are then fed into the decoder. After that, an error detection check is carried out to check on the multiplexed data correctness. Successful detection is fed back to the transmitter through an ACK over a low rate feedback channel that is assumed to be error free. This ACK flags a correct detection indicating that no further processing is required. Upon receiving a NACK due to false error check, the transmitter triggers the first diversity column. After reception, this diversity column is combined with the $P_{s} / n_{t}$ basic columns. The mixed mode basic-diversity symbols are then re-decoded and re-checked for errors. Another NACK triggers the diversity encoding of the next $n_{t}$ symbols in the packet. This process is iteratively repeated until a positive ACK is received, or full diversity capabilities of the transmitter are reached, i.e. when all $P_{s} / n_{t}$ diversity columns are transmitted. If all diversity columns are transmitted, while a NACK is flagged, the algorithm halts and an erroneous packet is presumed.

The key advantage of the proposed scheme is the ultimate adaptation to the channel quality. The RAID scheme adaptively switches between the three schemes described previously in a gradual manner. This incremental transition guarantees the reliability of the diversity mode while gradually boosting the SE which is given by

$$
S E=\frac{m P_{s}}{P_{s} / n_{t}+\text { iterations }} \text { bits } / \mathrm{s} / \mathrm{Hz}
$$

where $m$ is number of bits per modulated symbol, and iterations is number of processed diversity columns.

\subsection{Energy-Conservative Incremental Diversity (ECID)}

Typically, broadcast systems are characterized by open loop communication. Accordingly, within a broadcast MIMO environment, the multiplexing/diversity configuration should be selected to suffice the QoS requirements of receivers under inferior channel conditions. This might oblige receivers under good channel conditions to commit unnecessary energy resources in decoding and processing of the redundant data that has been transmitted by the broadcasting station to enrich the reliability of the transmission for inferior receivers within the broadcast network. Accordingly, in this paper, the proposed incremental diversity framework is also customized for an energy-conservative mode in which each receiver independently limits the number of decoded and processed broadcast symbols in accordance to its instantaneous channel conditions within the broadcast MIMO network.

To the best of the author's knowledge, this work is the first to deal with the SM/STBC switching from an energy conservation context within a broadcast environment. The main advantage of the proposed energy-conservative incremental diversity (ECID) is that it operates over the symbol-level. Packet-level approaches relying on fountain codes for adapting to broadcast MIMO environments require the receivers to continue reception of packets 


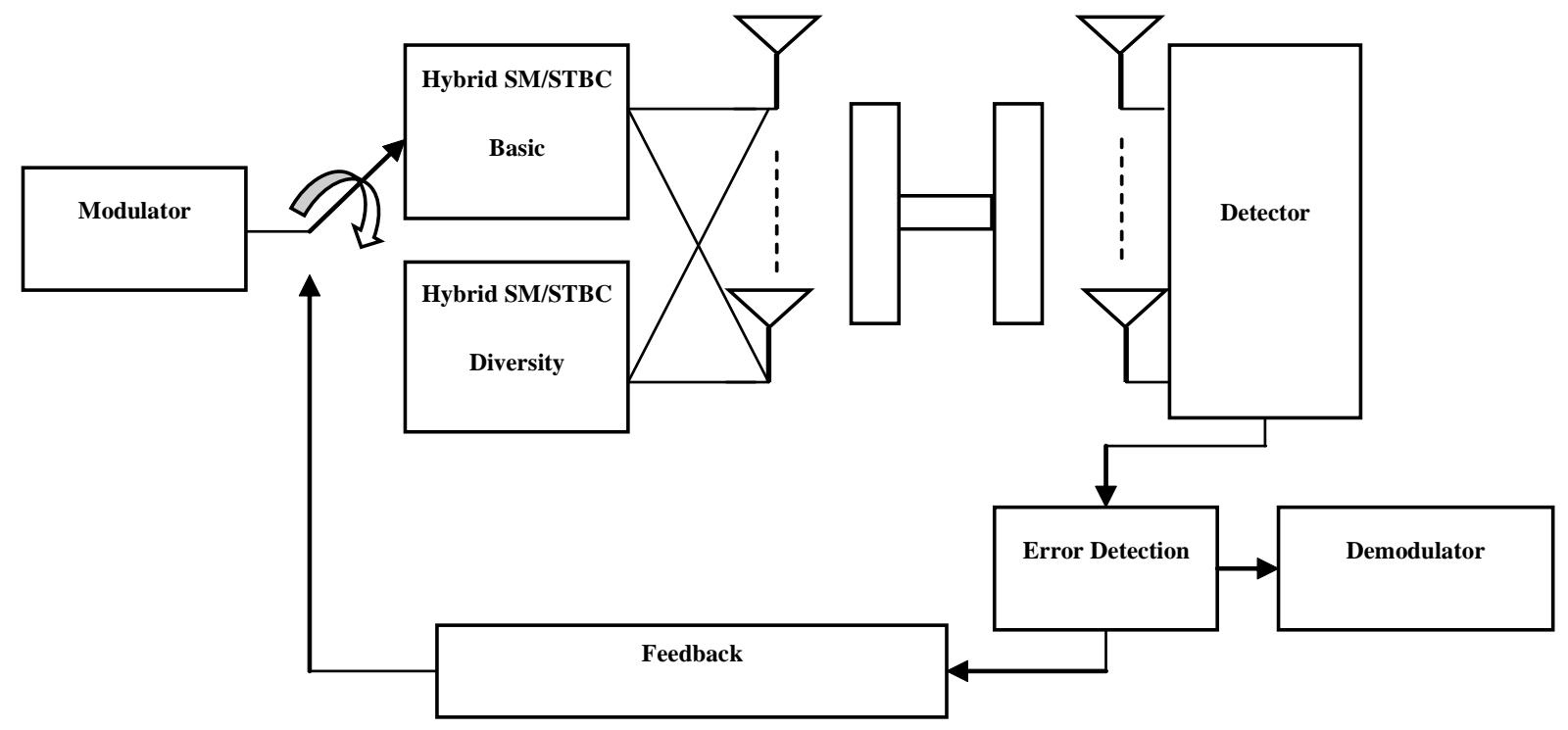

Figure 1. System model of rate-adaptive incremental diversity.

transmitted by the broadcast station until a certain number of correctly decoded packets are available for composite decoding [16]. Within such process, packets that fail error detection checks are ignored and therefore, constitute a source of waste of the receiver's energy resources. The proposed ECID symbol-level approach renders the possibility for superior energy conservation capabilities of the receiver by limiting the number of received symbol to those that would help a given packet pass the error detection check.

Figure 2 presents the system model of the proposed ECID scheme for a MIMO system with $n_{t}$ Tx and $n_{r}$ Rx antennas. Similar to the encoding of the RAID scheme, the Hybrid encoding procedure is carried out over two encoding phases; basic and diversity. When processing a packet of size $P_{s}$ modulated symbols, each encoder takes in $n_{t}$ modulated symbols at a time. The ECID algorithm does not have a feedback channel that links the receiver to the transmitter. Consequently, the incremental diversity concept is not applied on a transmission-reception basis. It instead takes an incremental detection approach that is controlled independently by each receiver within the broadcast environment. Thus, unlike the RAID algorithm, both the basic and diversity vectors are simultaneously transmitted giving a fixed rate transmission. According to the receiver's experienced channel quality, the receiver decides on the amount of diversity vectors required for successful detection.

In this sense, a mixer takes in the basic and diversity vectors from the encoders, and concatenates them in a composite transmission matrix. Unlike the conventional hybrid SM/STBC encoder, the basic and diversity columns are not located alternately. Instead, they are bulkily positioned, where a bulk of the $n_{t} \times P_{s} / n_{t}$ basic vectors is placed next to the bulk of $n_{t} \times P_{s} / n_{t}$ diversity vectors. The organization of the transmission matrix such that the basic columns are the first to be received is mandatory for the efficient application of the proposed scheme. As discussed formerly, the incremental approach resides in controlling the amount of diversity columns required for successful detection. Thus once redeemed successful, the receiver front end could enter into a sleep mode to avoid redundant energy drain inherited in reception and/or processing of unwanted diversity vectors.

The key challenge of the ECID algorithm is the termination of reception of the diversity symbols once an ACK is received. This requires the assumption of the processing rate being higher than the reception rate. Hence, once the detection is redeemed successful, the receiver front end goes in to sleep mode and no symbols are further received. Alternatively, a buffer could be deployed between the receiver front end and the decoder. This buffer allows the storage of the diversity symbols that are received before the ACK instigation.

The percentage of energy conservation in the ECID mode is a function of the number of diversity columns after which the detection is redeemed successful. This energy conservation is a computed with respect to the energy consumption of the full diversity mode and is given by

$$
E C=\left(\frac{1}{2}-\frac{\text { iterations } n_{t}}{2 P_{s}}\right) \times 100 \%
$$




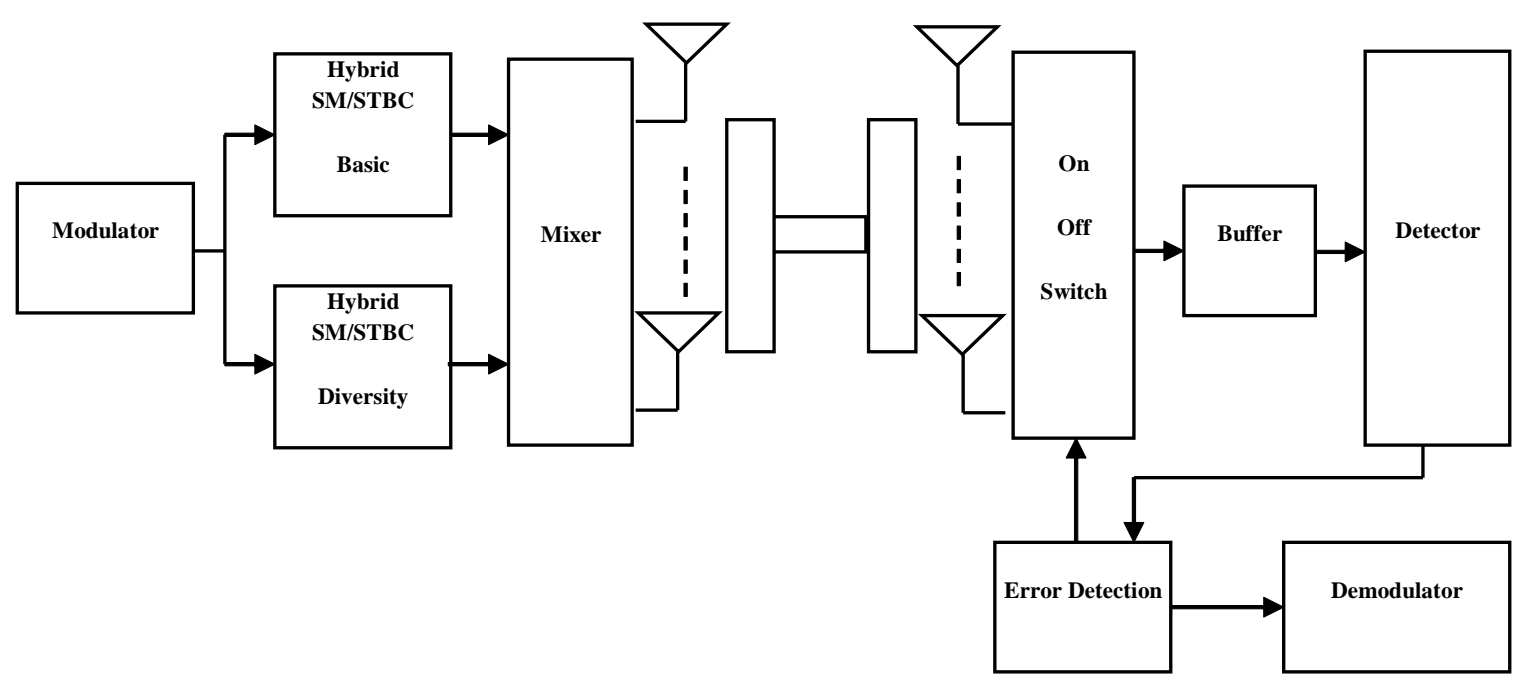

Figure 2. System model of energy-conservative incremental diversity.

As the number of required diversity columns traverses the interval of $P_{s} / n_{t}$ to 0 , the percentage of reception energy conservation evolves from $0 \%$ up to $50 \%$. In short, the proposed ECID scheme allows the receiver to go to sleep mode up to $50 \%$ of the reception time when compared to full diversity operation. Thus, it may help enhance the battery lifetime which is a crucial issue in wireless networks.

\section{Simulation Results}

In this section, performance of proposed RAID and ECID schemes is evaluated and compared to the conventional SM and STBC schemes. The comparison is viewed from the perspective of Bit Error Rate (BER), required decoding complexity, and the attainable SE or the percentage of EC in case of RAID or ECID, respectively. The switching endpoints are SM, STBC, and Hybrid SM/STBC with $N$ Alamouti STBC blocks. Simulations are carried out for different infrastructure of $2 \times 2,4 \times 4$, and $8 \times 8$ MIMO systems. As mentioned, Alamouti STBC is the only orthogonal STBC scheme that is full rate. Therefore, Alamouti STBC is the sole STBC scheme adopted throughout the upcoming simulations. This means that higher order MIMO systems of 4 and 8 antennas are deployed with Hybrid SM/STBC with 2 and 4 Alamouti STBC blocks, respectively.

All the simulated MIMO schemes including SM, STBC, Hybrid SM/STBC, RAID and ECID are decoded with the modified sphere decoding (SD) that is proposed in [17]. The modified SD guarantees the maximum likelihood (ML) solution at a dramatically lower decoding complexity. The metric used to measure the decoding complexity in [17] is the number of Floating Point Operations per Second (FLOPS). However, the FLOPS function was eliminated from MATLAB because of its inaccuracy [18]. As an alternative, the number of visited nodes in the tree search of the modified SD is used in this paper. In order to compare the SD complexity for different modulation sizes, the number of visited nodes is normalized by the number of transmitted bits. Hence, the nodes metric herein represents the number of nodes required to decode 1 information bit.

Simulations are carried out over a stream of $10^{6}$ information bits. The MIMO schemes are compared for different QAM constellation sizes with 3 different packet sizes each. Simulation results are plotted versus SNR that is defined as the ratio of total transmit power to the noise variance per receive antenna. The space time symbols are normalized by $1 / \sqrt{E_{s} n_{t}}$ to have unit energy where $E_{s}$ is the average energy per modulated symbol. The Rayleigh channel is assumed to be independent and identically distributed (i.i.d) frequency flat slowly varying with complex fading coefficients. The fading coefficients remain fixed over a block of $2 P_{s} / n_{t}$ and are drawn independently in every block. Perfect CSI is assumed at the receiver but not at the transmitter side. The additive white Gaussian noise is a complex Gaussian variable of zero mean and variance $N_{0} / 2$.

\subsection{Performance of ID}

Figure 3(a) shows the BER of a MIMO system with $2 \mathrm{Tx}$, and $2 \mathrm{Rx}$ antennas of the proposed ID scheme compared to SM, and Alamouti STBC schemes with 16 QAM and a packet size of 4 symbols. As shown in the fig- 


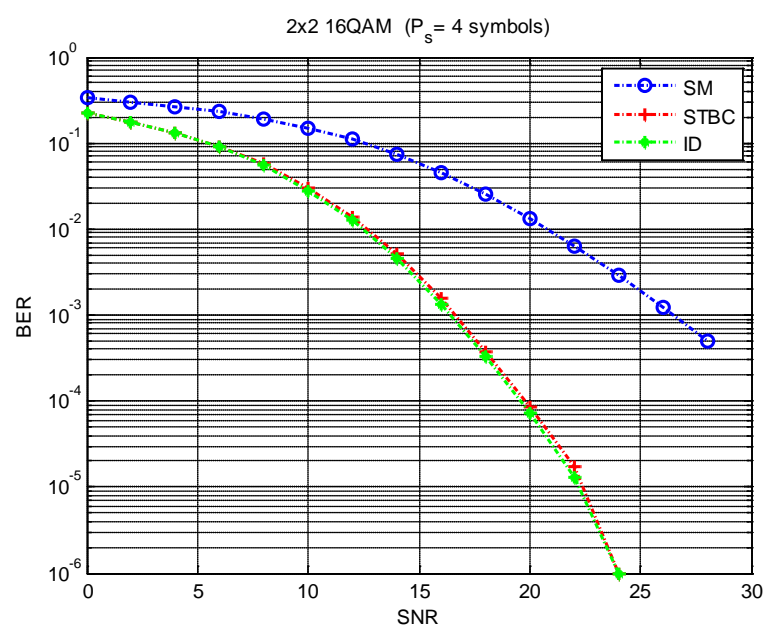

(a)

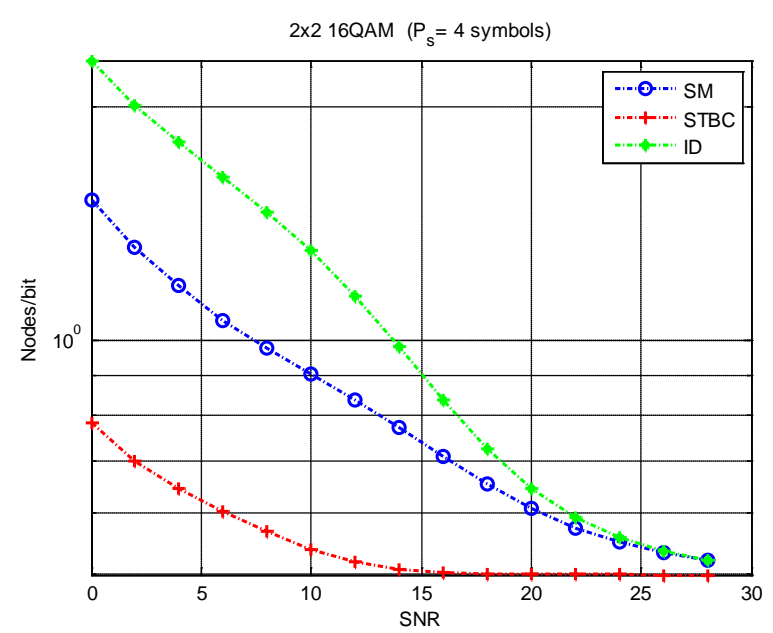

(b)

Figure 3. (a) BER of $2 \times 2$ MIMO, 16 QAM, $P_{s}=4$ Symbols; (b) Decoding Complexity of $2 \times 2$ MIMO, 16 QAM, $P_{s}=4$ Symbols.

ure, the BER curve of the proposed ID scheme coincides with that of the Alamouti scheme with a drastic gap from that of the SM. Looking at the decoding complexity in Figure 3(b), the average number of visited nodes of ID is relatively higher than SM and STBC approaching to that of SM as SNR increases. It is also to be noted that both RAID and ECID share the same BER and decoding complexity performance measures.

Figure 4(a) shows the SE of the proposed ID scheme operating in the RAID mode. The SE curves of SM, and STBC are eliminated from the figure as they remain constant independent of SNR. The SE of the RAID scheme has a lower bound of $4 \mathrm{~b} / \mathrm{s} / \mathrm{Hz}$, which is the same as that of the STBC with a 16 QAM constellation. As the SNR increases, the SE keeps on increasing till it reaches the maximum attainable value of $8 \mathrm{~b} / \mathrm{s} / \mathrm{Hz}$ which could have been reached if a pure SM mode was deployed. The corresponding EC percentage resulting from adopting the ECID scheme is plotted in Figure 4(b). As depicted in the figure, the ECID scheme experiences a gradual enhancement in the reception energy consumption in correspondence to increasing SNR. It is to be noted that simulations have also been extended to an $8 \times 8$ MIMO system with a 16 QAM constellation. Within such configuration, the proposed ID scheme switches from SM, towards Hybrid SM/STBC that comprises 4 Alamouti blocks. The packet size is set to 16 modulated symbols. The figure results have been left out due to lack of space. However, it is to be reported that the performance of the $8 \times 8$ ID scheme has reflected the same trends as the case of $2 \times 2$ ID with SE ranging from $16 \mathrm{~b} / \mathrm{s} / \mathrm{Hz}$ to $32 \mathrm{~b} / \mathrm{s} / \mathrm{Hz}$ which are the corresponding SE of STBC and $\mathrm{SM}$, respectively. The EC has displayed steeper increase with SNR compared to the $2 \times 2$ ID case.

\subsection{Performance Comparison for Different Packet Sizes}

In order to investigate the effect of the packet size on the performance of the ID scheme, the simulation of the $2 \times 2$ MIMO with 16 QAM is carried out for a packet size of 4, 8, and 16 modulated symbols. The BER and decoding complexity of the 8 and 16 symbol packets show the same trends as the 4 symbol packet that was discussed previously. The packet size has no effect on the BER and the decoding complexity. However, it affects the SE and the EC of the RAID and ECID schemes, respectively.

Figure 5(a) compares the SE of the $2 \times 2$ RAID scheme for the different packet sizes. The SE curves show that processing packets of smaller number of symbols leads to higher SE at a given SNR. The figure shows a 1 $\mathrm{dB}$ and $2 \mathrm{~dB}$ superiority of the 4 symbol packet to the 8 and 16 symbol packets, respectively. However, this gain reduces relatively at low and high SNR extremes. Similarly, the EC percentage of the three different packet sizes is compared in Figure 5(b). The results show that smaller packet sizes have higher potential to energy conservation.

\subsection{Performance Comparison for Different Packet Sizes}

In this section, the effect of the number of antennas on the performance of the ID scheme is explored. The ID 


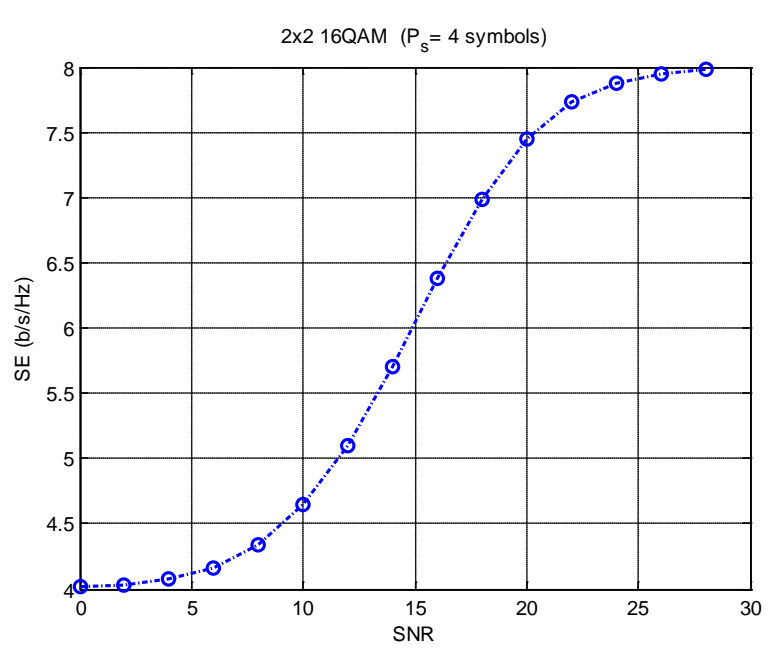

(a)

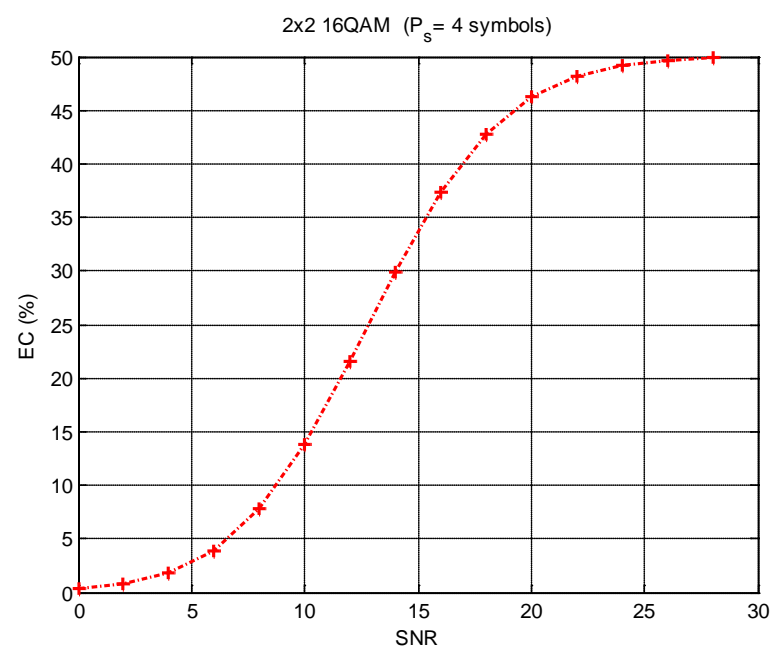

(b)

Figure 4. (a) SE of $2 \times 2$ MIMO, 16 QAM, $P_{s}=4$ Symbols; (b) EC of $2 \times 2$ MIMO, 16 QAM, $P_{s}=4$ Symbols.

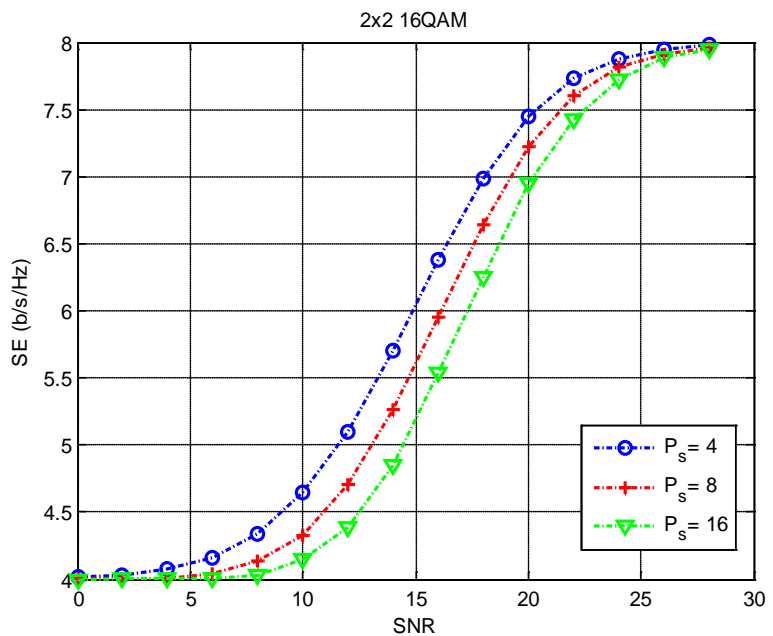

(a)

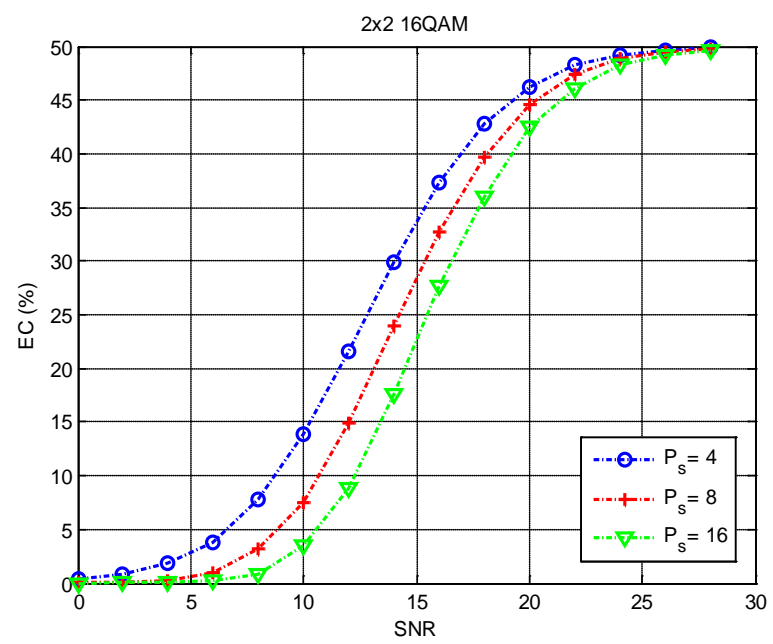

(b)

Figure 5. (a) SE of $2 \times 2$ RAID MIMO, 16 QAM for Different $P_{s}$; (b) EC of $2 \times 2$ RAID MIMO, 16 QAM for Different $P_{s}$.

scheme is simulated for different antenna arrays and different constellation sizes. Figure 6(a) compares the BER performance of $2 \times 2$ and $4 \times 4$ ID with 16 and 4 QAM constellations, respectively, over a channel that remains fixed over 16 symbol durations. Thus, the packet size of the $2 \times 2$ and the $4 \times 4$ ID is set to 16 and 32 symbols, respectively. The $2 \times 2$ system operates with higher constellation size so as to compensate for the SE loss inherited in deploying less number of antennas in comparison to the $4 \times 4$ system. As shown in the figure, the $4 \times 4$ system significantly outperforms the $2 \times 2$ one. The decoding complexity of the two systems displayed in Figure 6(b) shows that the $4 \times 4$ ID has a higher decoding complexity. However, the complexity difference becomes tolerable as the SNR increases. Figure 7(a), and Figure 7(b) display the SE and EC, respectively, of the two systems. The results for SE and EC show that $4 \times 4$ system outperforms the $2 \times 2$ system varies from around $6 \mathrm{~dB}$ up to $10 \mathrm{~dB}$ at lower and higher SNR levels respectively.

\subsection{Performance Comparison for Different Modulation Constellations}

In this section, the performance of the proposed ID scheme is analyzed for different modulation constellation sizes. Unlike the previous analysis, the infrastructure is maintained fixed. Thus, the switching endpoints experience different SE in correspondence to changing the constellation size. Figure 8(a), Figure 8(b), and Figure 10(a) 


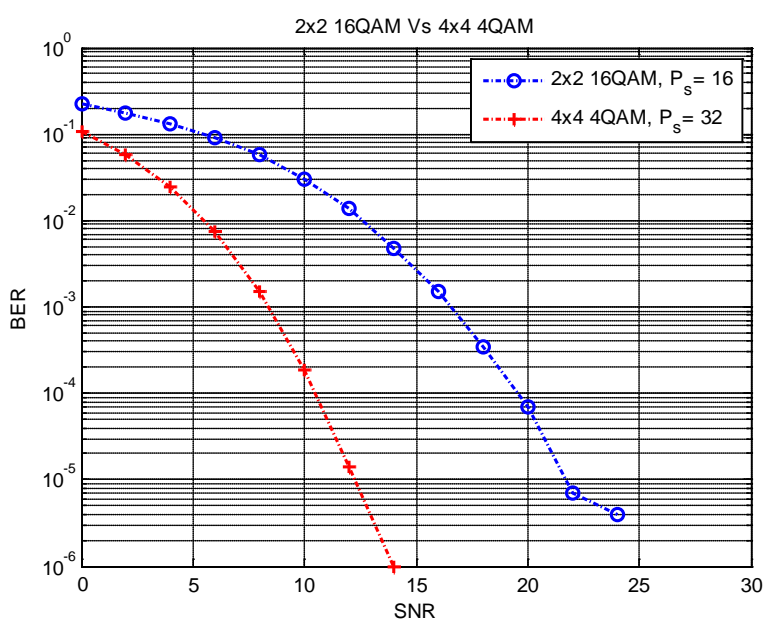

(a)

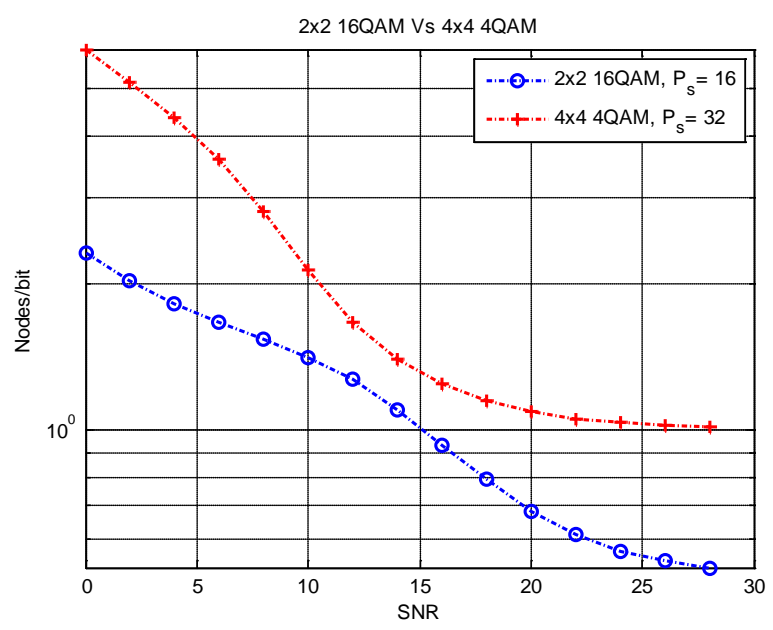

(b)

Figure 6. (a) BER of $2 \times 2$ ID, 16 QAM Vs $4 \times 4$ ID, 4 QAM; (b) Decoding Complexity of $2 \times 2$ ID, 16 QAM Vs $4 \times 4$ ID, 4 QAM.

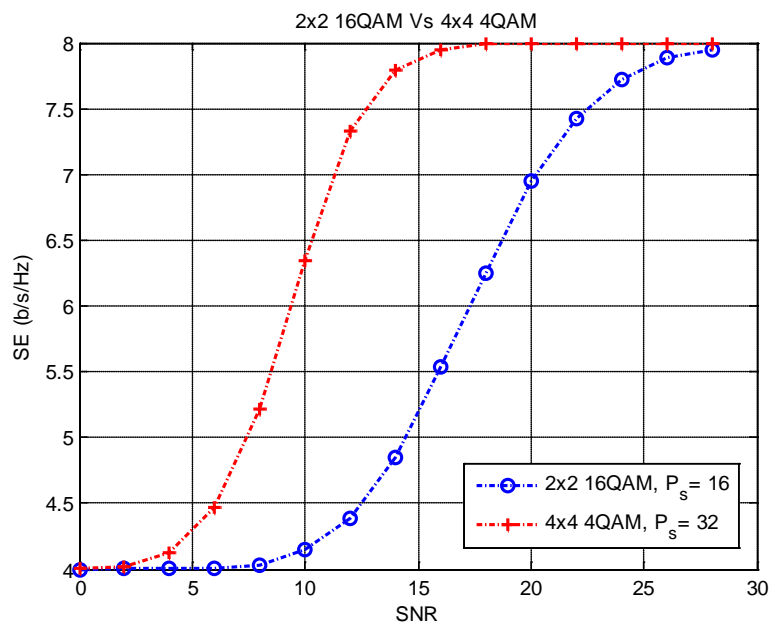

(a)

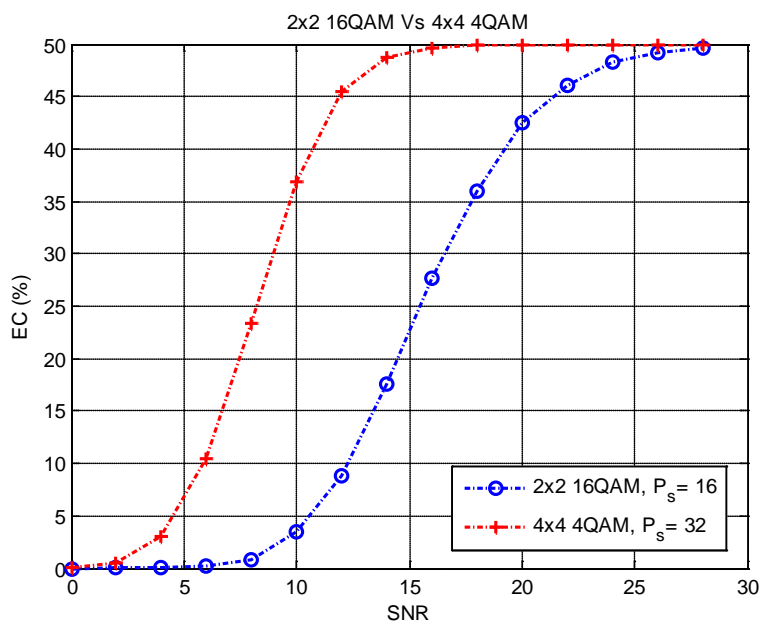

(b)

Figure 7. (a) SE of $2 \times 2$ RAID, 16 QAM Vs $4 \times 4$ RAID, 4 QAM; (b) EC of $2 \times 2$ ECID, 16 QAM Vs $4 \times 4$ ECID, 4 QAM.

show the BER, decoding complexity, and SE, respectively, of a $2 \times 2$ MIMO system with 8 symbol packet simulated for 4, 16, and 64 QAM. For a fair comparison of the EC, the infrastructure should adaptively change with the constellation size as was illustrated in the previous section. Thus, the EC curves are omitted due to their incompliance with the analysis herein. Comparison of BER performance of the three constellation sizes is quite intuitive where smaller constellations achieve better BER performance due to the larger Euclidean distance.

The noteworthy remark lies in the rise up trend of the SE curves of the different constellation sizes. As shown in Figure 10(a), the SE of higher order QAM constellation spans a wider range of SNR to evolve from its lower bound than lower order constellation sizes. This gives the chance to the lower order constellation sizes to catch up with the SE of the higher order ones at certain SNR ranges. For example, at the SNR of $10 \mathrm{~dB}$, the SE of 4 QAM is $3.41 \mathrm{~b} / \mathrm{s} / \mathrm{Hz}$ while that of the 16 QAM is $4.32 \mathrm{~b} / \mathrm{s} / \mathrm{Hz}$. Meanwhile, at the same SNR, the BER of the 4 QAM is $8 \times 10^{-4}$ in comparison to the $3 \times 10^{-2}$ BER of the 16 QAM constellation. This means that, at this SNR value, sacrificing a SE of $0.9 \mathrm{~b} / \mathrm{s} / \mathrm{Hz}$ results in a significant enhancement in the BER. Thus, at this SNR value, the 4 QAM might be favored to the 16 QAM constellation. Moreover, the 16 QAM gives approximately the same SE as the $64 \mathrm{QAM}$ at the SNR of $18 \mathrm{~dB}$ with a drastic improvement in the BER performance.

The complexity curves shown in Figure 8(b) show that at the SNR of $10 \mathrm{~dB}$, the decoding complexity of 4 QAM is the same as that of 16 QAM. Moreover, the decoding complexity of 16 QAM is even lower than that of 


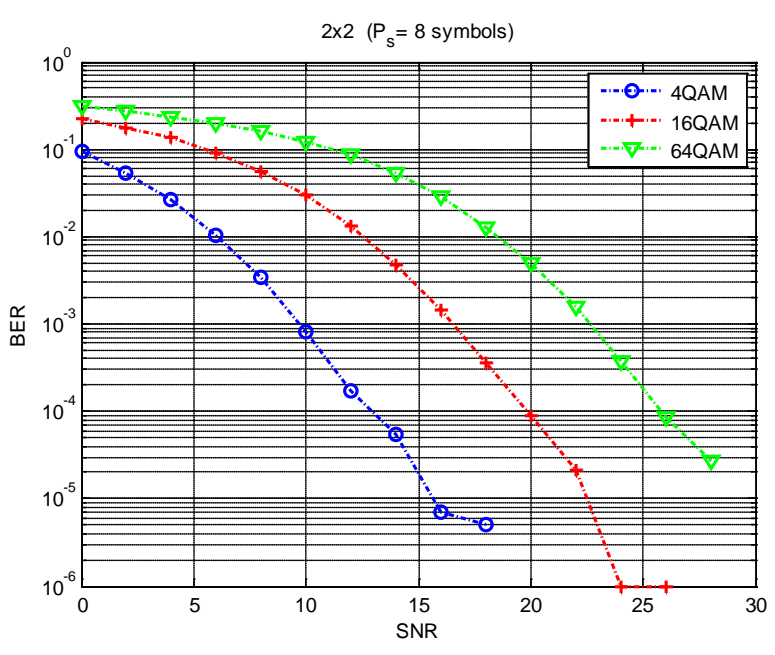

(a)

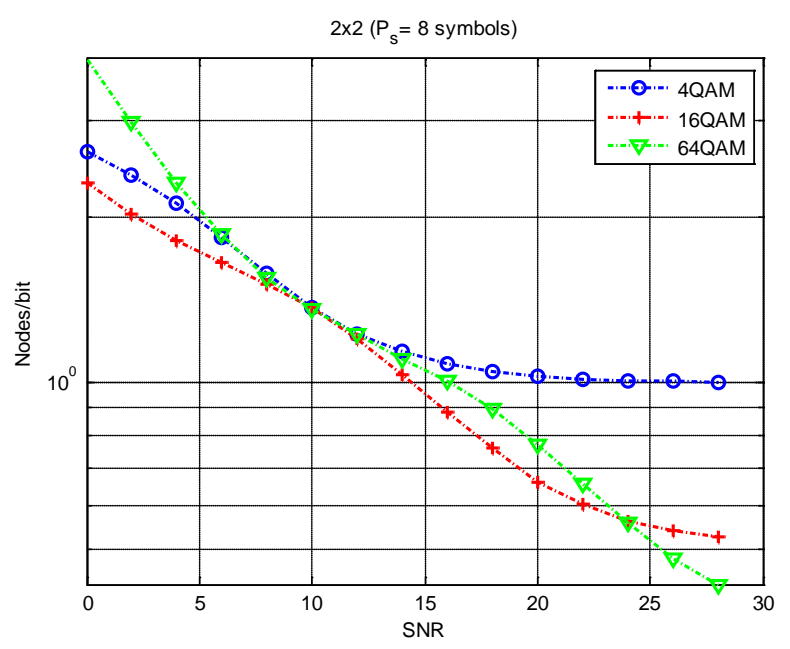

(b)

Figure 8. (a) BER of $2 \times 2$ ID, $P_{s}=8$ for Different Constellation; (b) Decoding Complexity of $2 \times 2$ ID, $P_{s}=8$ for Different Constellation.

64 QAM at SNR of $18 \mathrm{~dB}$. This consolidates with the idea of favoring 4 QAM over 16 QAM and 16 QAM over 64 QAM at SNR of 10 and $18 \mathrm{~dB}$, respectively. This results in a drastic improvement in the BER along with a potential to minimizing the decoding complexity at the price of a tolerable to negligible reduction in the SE.

As an extension to the previous analysis, the simulation is extended to a $4 \times 4$ RAID scheme with 4, 16 and 64 QAM and a 16 symbol packet. The BER, decoding complexity, and SE curves are shown in Figure 9(a), Figure 9(b), and Figure 10(b), respectively. Again the BER curves show a great superiority of the lower order constellation sizes to the higher order ones. For the conventional multiplexing and diversity scheme, this BER enhancement comes at the expense of the SE. However, the gradual enhancement in the SE inherited in the deployment of the RAID scheme alters the tradeoff. Comparing 4 QAM and 16 QAM constellations at the SNR of $12 \mathrm{~dB}$ shows that adopting 4 QAM enhances the BER performance by more than two orders of magnitude over 16 QAM. Moreover, at this SNR value, 4 QAM experiences half the decoding complexity of 16 QAM. The price it pays in return is $1.3 \mathrm{~b} / \mathrm{s} / \mathrm{Hz}$ loss in the SE that is compromised with the significant enhancement in both the BER and decoding complexity. The comparison of 16 and 64 QAM yields significant remarks. For the SNR range of $18-22 \mathrm{~dB}$, the SE of 16 QAM is even higher than that of 64 QAM. Its decoding complexity is almost one third that of 64 QAM, whereas its BER performance is superior by more than two orders of magnitude.

\section{Conclusion and Future Work}

In this paper, an incremental diversity scheme is proposed. It incrementally switches from SM towards STBC or Hybrid SM/STBC. Two modes of operation for the ID schemes are proposed to be chosen according to the system specifications. The first mode is the RAID scheme that aims at boosting the SE of the MIMO system. Alternatively, the ECID scheme maintains the SE of the conventional diversity scheme while rendering the opportunity for receivers under superior channel conditions within a broadcast network to minimize the number of received symbols and enter into an energy-conserving sleep mode until the next packet is to be received. Results show that processing packets with smaller number of symbols, the RAID and ECID schemes give higher tendency to enhanced levels of SE or EC, respectively. The noteworthy remark is that obtaining higher levels of SE with either the RAID or ECID schemes is better achieved by going for higher order antenna array rather than larger modulation constellation size. Moreover, comparing performance of the RAID scheme with different constellation sizes for the same infrastructure gives a very important remark. At certain SNR ranges, SE of lower order constellation sizes are very close to those of higher order ones and might also surpass them. On the other hand, lower order constellation sizes drastically outperform higher order ones in the BER performance along with a significant reduction in the decoding complexity. This implies that at some SNR ranges, tolerating a minor degradation in the $\mathrm{SE}$, leads to significant enhancement in the BER performance and decoding complexity.

In future work, the performance of the proposed system can be enhanced further by computing soft reliability 


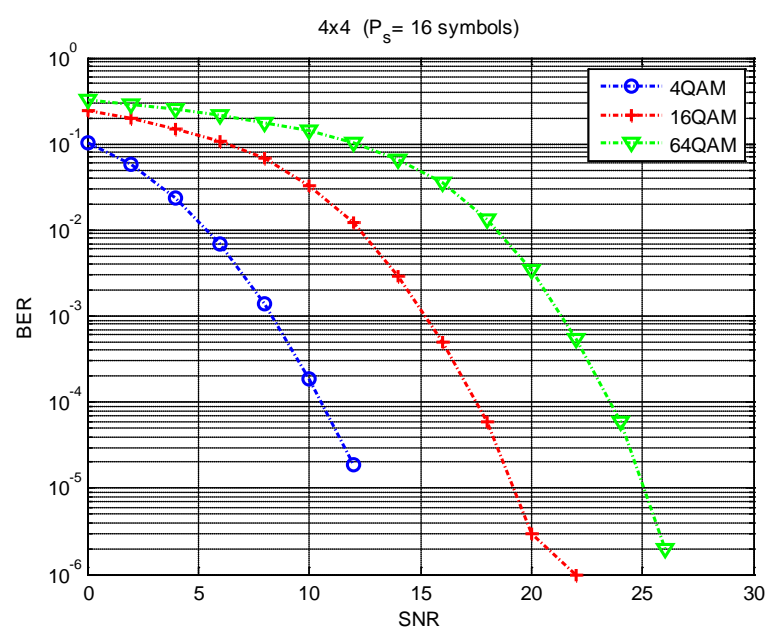

(a)

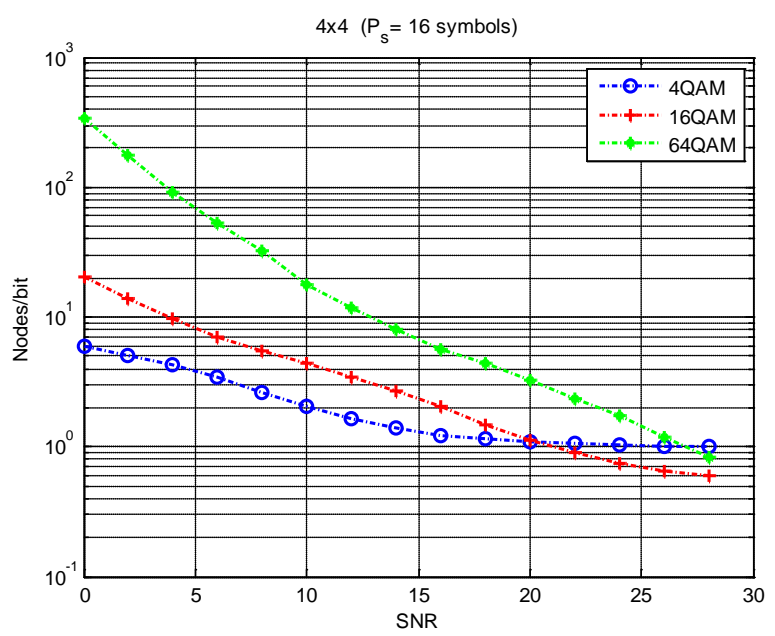

(b)

Figure 9. (a) BER of $4 \times 4$ ID, $P_{s}=16$ for Different Constellation; (b) Decoding Complexity of $4 \times 4$ ID, $P_{s}=16$ for Different Constellation.

$2 \times 2 \quad\left(P_{s}=8\right.$ symbols $)$

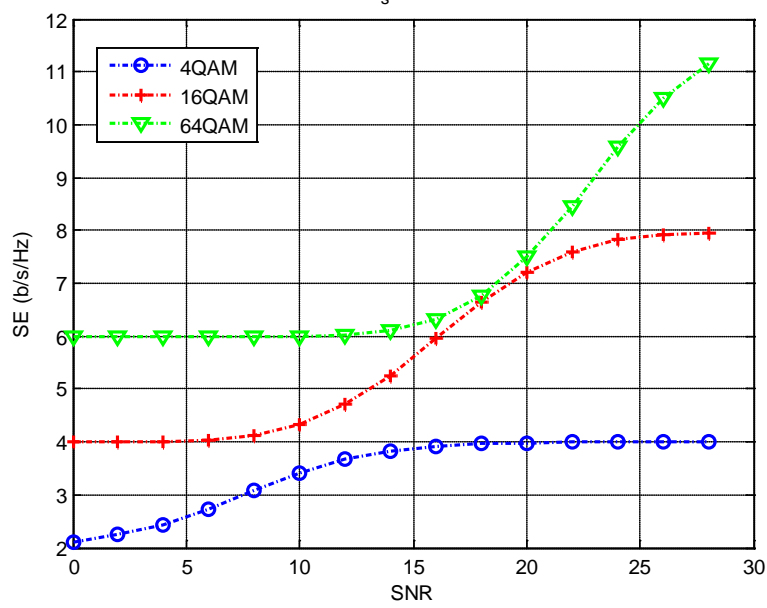

(a)
$4 \times 4 \quad\left(P_{s}=16\right.$ symbols $)$

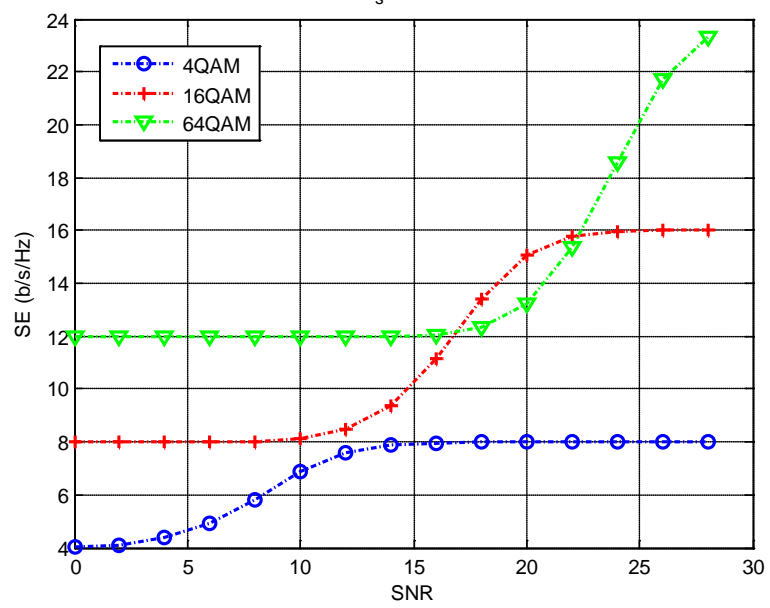

(b)

Figure 10. (a) SE of $2 \times 2$ ID, $P_{s}=8$ for Different Constellation; (b) SE of $4 \times 4$ ID, $P_{s}=16$ for Different Constellation.

information at the receiver side. This information can be used as an indicator to the modulation size to be used, the initial space time scheme to start the transmission with, and the amount of redundancy required per retransmission.

\section{References}

[1] Vucetic, B. and Yuan, J. (2003) Space-Time Coding, Wiley. http://dx.doi.org/10.1002/047001413X

[2] Tse, D. and Viswanath, P. (2005) Fundamentals of Wireless Communications. Cambridge.

[3] Chae, C., Katz, M., Suh, C. and Jeong, H. (2004) Adaptive Spatial Modulation for MIMO-OFDM. IEEE Wireless Communications and Networking Conference, 1, 87-92.

[4] Lopez-Vicario, J. and Anton-Haro, C. (2005) Adaptive Switching between Spatial Diversity and Multiplexing: A Cross-Layer Approach. IST Mobile Communications Summit, June 2005.

[5] Lozano, A. and Jindal, N. (2010) Transmit Diversity vs. Spatial Multiplexing in Modern MIMO Systems. IEEE Transactions on Wireless Communications, 9, 186-197. http://dx.doi.org/10.1109/TWC.2010.01.081381

[6] Heath, R.W. and Paulraj, A.J. (2005) Switching between Diversity and Multiplexing in MIMO Systems. IEEE Trans- 
actions on Communications, 53, 962-968. http://dx.doi.org/10.1109/TCOMM.2005.849774

[7] Choi, Y. and Alamouti, S.M. (2008) A Pragmatic PHY Abstraction Technique for Link Adaptation and MIMO Switching. IEEE Journal on Selected Areas in Communications, 26, 960-971. http://dx.doi.org/10.1109/JSAC.2008.080812

[8] Wu, Y. and Tellambura, C. (2004) Low-Complexity Optimal Detection for Hybrid Space-Time Block Coding and Spatial Multiplexing. Vehicular Technology Conference, Montreal, 25-28 September 2006, 1-4.

[9] Elshabrawy, T. and Elsharkawy, M. (2011) Computationally Efficient Implementation for Incremental Redundancy within Wireless Networks. IEEE International Symposium on Personal Indoor and Mobile Radio Communications, Toronto, 11-14 September 2011, 1051-1055.

[10] El-Shabrawy, T. and Wahed, S.A. (2009) Adaptive Modulation and Coding for Broadcast DVB-H Systems. International Symposium on Personal, Indoor and Mobile Radio Communications, Tokyo, 13-16 September 2009, 1292-1296.

[11] Moreira, J.C. and Farrell, P.G. (2006) Essentials of Error-Control Coding. John Wiley \& Sons Ltd., Chippenham, 6576.

[12] Elshabrawy, T. and Nafae, S. (2013) An Adaptive MIMO System Using Incremental Diversity. Vehicular Technology Conference 2013, Las Vagas, 2-5 September 2013, 1-5.

[13] Larsson, E.G. and Stoica, P. (2005) Space-Time Block Coding for Wireless Communications, Cambridge.

[14] Goldsmith, A. Multiple Antennas and Space-Time Communications in Wireless Communications, Cambridge, 321-347.

[15] Hwang, K.J., Lee, S. and Chang, K. (2003) Adaptive Rate MIMO System Using Space-Time Block Mapping. The 57th IEEE Semiannual Vehicular Technology Conference, 1, 774-778.

[16] Sheikh, M.U., Jagusz, R. and Lempiäinen, J. (2011) Performance Evaluation of Adaptive MIMO Switching in Long Term Evolution. International Wireless Communications and Mobile Computing Conference, Istanbul, 4-8 July 2011, 866-870.

[17] Chan, A.M. and Lee, I. (2002) A New Reduced-Complexity Sphere Decoder for Multiple Antenna Systems. IEEE International Conference on Communications, 1, 460-464.

[18] Minka, T. (2011) Microsoft Corporation. http://research.microsoft.com/en-us/um/people/minka/software/lightspeed/ 
Scientific Research Publishing (SCIRP) is one of the largest Open Access journal publishers. It is currently publishing more than 200 open access, online, peer-reviewed journals covering a wide range of academic disciplines. SCIRP serves the worldwide academic communities and contributes to the progress and application of science with its publication.

Other selected journals from SCIRP are listed as below. Submit your manuscript to us via either submit@scirp.org or Online Submission Portal.
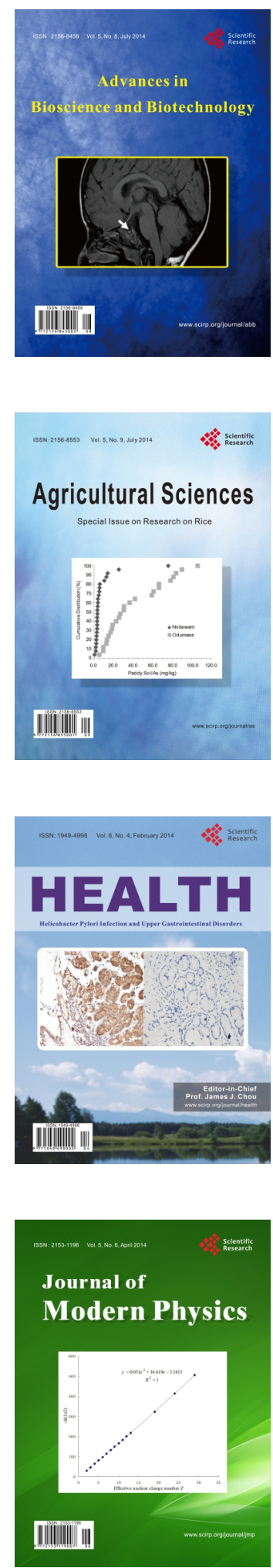
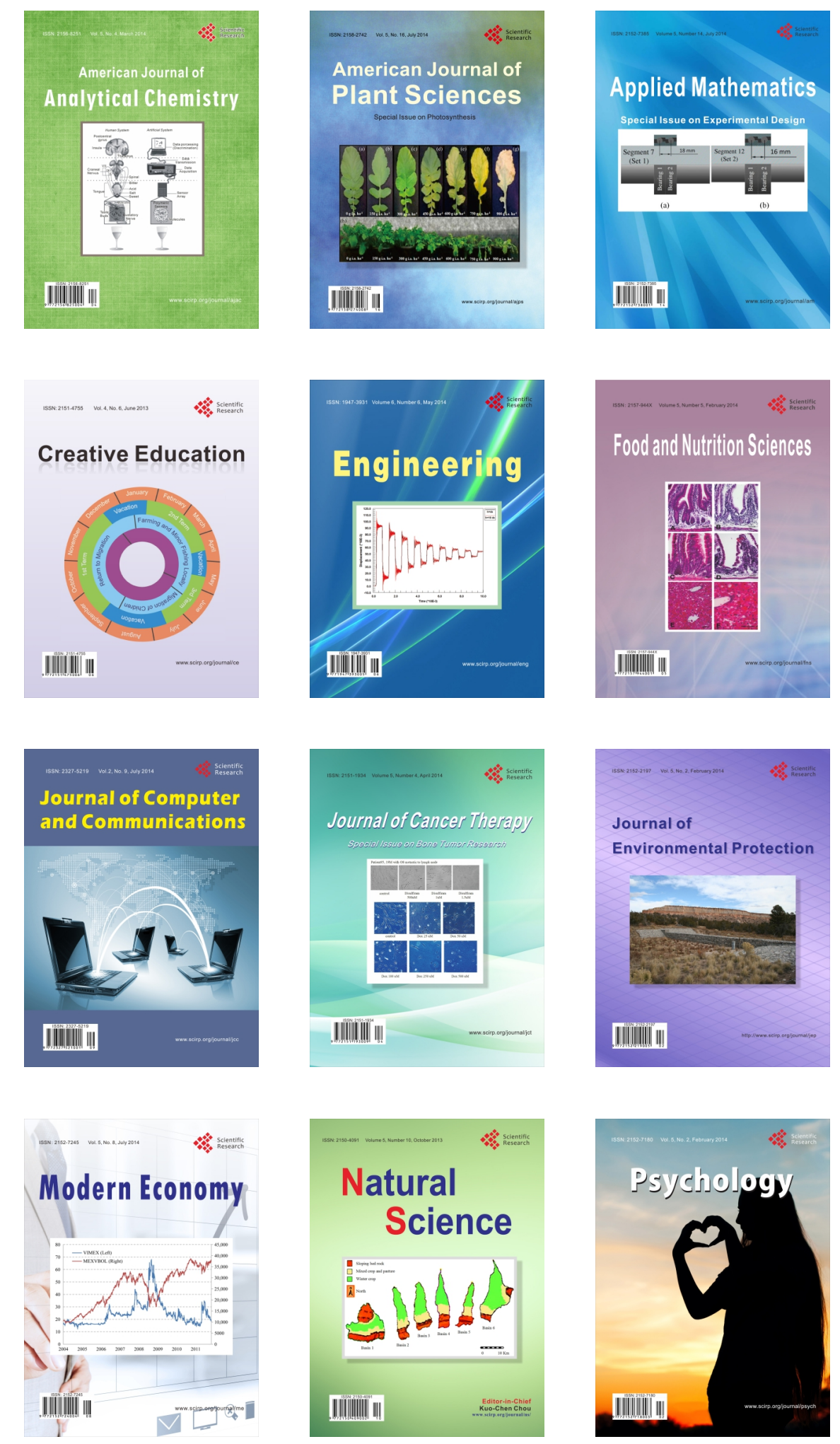